\title{
Edad, escolarización y tareas de Fluencia Verbal para el screening de pacientes con Enfermedad de Alzheimer
}

\author{
Adriana Goñi Sarriés ${ }^{1}$, José J. López-Goñi², David Granados-Rodríguez ${ }^{3}$ y Ángela González-Jiménez ${ }^{4}$ \\ 1 Servicio Navarro de Salud - Osasunbidea (España). \\ 2 Departamento de Psicología y Pedagogía. Universidad Pública de Navarra (España). \\ 3 Servicio Andaluz de Salud (España) \\ 4 Hospital San Juan de Dios (Pamplona, España)
}

\begin{abstract}
Resumen: Introducción. Las pruebas de Fluencia Verbal (FV) Semántica (FVS) y Fonológica (FVF) son muy empleadas en la práctica clínica. Disponer de diferentes pruebas alternativas que además tengan en cuenta el efecto de variables sociodemográficas mejorarían su uso como tests de cribado, permitiendo diferenciar a personas con y sin Enfermedad de Alzhei mer (EA). Objetivos. Comparar la capacidad discriminativa de las tareas de FVS "cosas en una casa" y "alimentos" frente a la tarea "animales" entre pacientes con EA $(n=50)$ y sujetos sanos $(n=50)$; comparar el uso de los fonemas "P"-"M"-"R" como tarea alternativa y/o paralela a los fonemas "F"-“A"-"S"; y valorar el uso combinado de ambos tipos de tareas junto con el de variables sociodemográficas para la discriminación de pacientes con EA y sujetos sanos. Resultados. Tanto la categoría semántica como fonológica muestran resultados semejantes, una alta correlación, mantienen la validez de criterio y permiten su utilización de forma paralela. El modelo de predicción que logra discriminar correctamente al 91\% de los sujetos evaluados es el que incluye las tareas "cosas en una casa", los fonemas "A" "S" y las variables edad y años de escolarización. Conclusiones. La utilización paralela de pruebas de FVS y FVF, junto con variables sociodemográficas mejora la capacidad discriminativa de las pruebas de FV.

Palabras-clave: Demencia; enfermedad de Alzheimer; cribado; exploración neuropsicológica; fluidez verbal; fluidez semántica; fluencia fonológica.
\end{abstract}

\section{Introducción}

La fluencia verbal (FV) es la capacidad de producir un habla espontáneamente fluida, sin pausas ni fallos en la búsqueda de palabras. A pesar de su aparente simplicidad es una tarea cognitiva compleja que requiere de la intervención de múltiples procesos cognitivos, como son los lingüísticos (denominación, vocabulario y velocidad de producción verbal), los mnésicos (memoria semántica y de trabajo) y los ejecutivos (estrategias de búsqueda, iniciación y supresión de respuestas dadas) (Rosser \& Hodges, 1994). También intervienen otras capacidades como la imaginación, la atención sostenida e iniciar una conducta en respuesta a una tarea novedosa (Kertesz \& Mohs, 1996).

En situaciones clínicas la FV se valora mediante distintas tareas en las que el sujeto debe generar tantas palabras como pueda bajo unas condiciones restringidas de búsqueda y en un tiempo limitado (normalmente un minuto) (Lezak, 1996; Spreen \& Strauss, 1998). Son tareas muy usadas en la valoración neuropsicológica (Ramírez, Ostrosky-Solís, OstroskySolís, Fernández, \& Ardila-Ardila, 2005) porque no necesitan instrumental, exigen poco tiempo, pueden realizarse en

* Dirección para correspondencia [Correspondence address]: José J. López Goñi. Departamento de Psicología y Pedagogía. Universidad Pública de Navarra. Campus de Arrosadía s/n 31006 Pamplona, Navarra. (España). E-mail: josejavier.lopez@unavarra.es
Title: Age, schooling and Verbal Fluency tasks for the screening of Alzheimer's disease patients.

Abstract: Introduction. The Verbal (VF), Semantic (SF) and Phonemic Fluency $(\mathrm{PF})$ tests are commonly used in clinical practice. Having different alternative tests, that could also allow for the effect of demographic variables, would improve their use as screening tests, making it possible to differentiate patients with or without Alzheimer's disease (AD). Aims. (1) To compare the discriminatory ability of the SF tasks "things in a house" and "food" versus the task "animals" among patients with the AD $(n=50)$ and healthy subjects $(n=50)$. (2) To compare the use of the phonemes such as "P", "M" and "R" as an alternative and/or parallel task to the phonemes "F", "A" and "S". (3) To assess the combined use of both tasks with the demographic variables for the screening of $\mathrm{AD}$ patients and the healthy ones. Results. Both semantic and phonemic categories indicate similar results, high correlation, support the criteria validity and allow for their use in a parallel way. Among all the different roles assessed, the most successful in screening correctly $91 \%$ of the evaluated subjects is the one that includes tasks such as "things in a house", the phonemes " $\mathrm{A}$ " and " $\mathrm{S}$ " and the age and schooling time variables. Conclusion. The parallel use of VF and PF, plus the demographic variables improve the discriminatory ability of the VF tests.

Keywords: Alzheimer's disease; dementia; screening; neuropsychological assessment; verbal fluency; semantic fluency; phonemic fluency.

cualquier medio y aplicarse incluso a personas no evaluables por otras pruebas (Carnero, 1999). Y es que, a pesar de que con la edad se observa una disminución del rendimiento, en general son tareas sensibles al daño cerebral (Alamo, Mir, Olivares, Barroso, \& Nieto, 1999; Galeote \& Peraita, 1999) en especial al deterioro cognitivo (Carnero, 1999)-, y a la enfermedad de Alzheimer (EA) (Alberca, Salas, Pérez-Gil, Lozano, \& Gil-Néciga, 1999; Cuetos-Vega, MenéndezGonzález, \& Calatayud-Noguera, 2007) por lo que sirven como test de cribado o rastreo cognitivo (Carnero-Pardo \& Lendínez-González, 1999).

Sus principales inconvenientes son la influencia de variables sociodemográficas (edad, sexo, nivel educativo) (Tombaugh, Kozak, \& Rees, 1999), culturales (Carnero-Pardo, 2000; Carnero-Pardo \& Del Ser, 2007; Carnero, 1999; PeñaCasanova, et al., 2009; Ramírez, et al., 2005; Steenhuis \& Ostbye, 1995) y de las circunstancias de la aplicación externas (temperatura de la sala, distracciones durante la prueba...) e internas (estado anímico, ansiedad, motivación...) que pueden afectar a la validez clínica de la prueba. Para mejorar su fiabilidad se ha propuesto un método estandarizado de aplicación que minimiza los efectos derivados de la administración y de la calificación de las pruebas (Ramírez, et al., 2005). En cuanto a la influencia del resto de variables se debe seguir con la investigación para determinar el influjo de las mismas. Además la mayoría de las baremaciones publicadas se han realizado con muestras extranjeras, por lo que es ne- 
cesario continuar con la investigación en situaciones clínicas de nuestro entorno.

Hay dos criterios de evocación en las pruebas de FV: el semántico o categorial y el fonológico. Cuando se emplean criterios semánticos (por ejemplo, "dígame cosas que puede encontrar en su casa") se denominan tareas de fluidez verbal semántica (FVS) y cuando se emplean criterios fonológicos (por ejemplo, "dígame palabras que empiezan por la letra "F") se denominan tareas de fluidez verbal fonológica (FVF).

Las tareas de FVS emplean habitualmente las categorías de "animales", "cosas que hay en una casa" (Fernández, et al., 2002), "frutas y vegetales", "utensilios de cocina" (PeñaCasanova, et al., 2009), "cosas que se pueden encontrar en un supermercado" (Garcés-Redondo, Santos, Pérez-Lázaro, \& Pascual-Millán, 2004) y "nombres de persona" (Sáez-Zea, Carnero-Pardo, \& Gurpegui, 2008). En esta misma línea, el set-test (Pascual Millán, et al., 1990) incluye cuatro categorías ("colores", "animales", "frutas" y "ciudades"), se ha validado en España y es aplicable a personas con baja escolaridad. La tarea "animales" ha sido validada en nuestro país y permite clasificar correctamente al $93 \%$ de los sujetos con demencia frente a los sanos (Carnero-Pardo \& Lendínez-González, 1999) aunque también se ha apreciado el efecto modulador de variables sociodemográficas (Carnero, et al., 2000; Lorente Aznar, et al., 2010), del que parece estar libre la categoría "nombres de persona" (Sáez-Zea, et al., 2008).

En cuanto a la evaluación de la FVF, los fonemas "F", "A" y "S" han sido los más utilizados en muestras anglosajonas ya que existe evidencia de que otros fonemas como "L" y "R" resultan más difíciles (Barry, Bates, \& Labouvie, 2008). En español se recomienda emplear los fonemas "P", "M" y "R" (Artiola, Hermosillo, Heaton, \& Pardee, 1999) que han sido normalizados con población española cognitivamente normal con edades comprendidas entre los 50 y 94 años (Peña-Casanova, et al., 2009). Ahora bien, los valores de sensibilidad para la demencia son bajos (44\%) (Canning, Leach, Stuss, Ngo, \& Black, 2004) y no existe una validación en población española con demencia.

En resumen, es importante continuar la búsqueda de datos neuropsicológicos normativos ya que la diversidad lingüística, cultural y social es cada vez mayor a la vez que es necesario reducir el efecto de variables (Contador, Fernández-Calvo, Ramos, Tapias-Merino, \& Bermejo-Pareja, 2010) como el nivel educativo y la edad sobre el rendimiento de las pruebas de FV para mejorar su uso como pruebas de cribado. Además, dado el amplio uso de estas pruebas es recomendable contar con pruebas paralelas válidas y contrastadas en la experiencia clínica (Fernández, et al., 2002).

Por todo ello, los objetivos de este estudio son: (1) comparar la capacidad discriminativa de las tareas de FVS "cosas en una casa" y "alimentos" frente a la tarea "animales" entre pacientes con EA y sujetos sanos; (2) comparar el uso de los fonemas "P", "M", y "R" como tarea alternativa y/o paralela a los fonemas " $F$ ", "A" y "S"; y (3) valorar el uso combinado de ambos tipos de tareas junto con el de variables socio- demográficas para la discriminación de pacientes con EA y sujetos sanos.

\section{Método}

\section{Participantes}

La muestra está formada por 100 sujetos, divididos en un grupo experimental y control. Los integrantes del grupo experimental son 50 pacientes de un Centro de Día Psicogeriátrico que viven con su familia y cumplen los criterios de EA probable según la clasificación CIE-10 (Organización Mundial de la Salud, 1992), con un deterioro cognitivo moderado. El grupo de control lo forman las parejas de los pacientes y cuidadores externos. Los criterios de admisión para el grupo experimental fueron: a) tener diagnóstico de EA; b) acudir al Centro de Día Psicogeriátrico; y c) tener más de 65 años. Para el grupo de control los criterios de exclusión fueron: a) obtener una puntuación menor de 23 en el MiniExamen Cognoscitivo (MEC); b) presentar un trastorno mental o una enfermedad neurológica.

En cuanto a las características sociodemográficas de la muestra, el 44\% de los sujetos del grupo de control $(n=22)$ son varones. Dicho grupo tiene una edad media de 76 años $(D T=7.7)$ y una media de 8.8 años de escolarización $(D T=$ 2.7). El grupo de pacientes con EA presenta un $50 \%$ de varones, una edad media de 72.5 años $(D T=7.2)$ y una media de 10.3 años de escolarización $(D T=2.5)$. Cuando se comparan ambos grupos, en dos ítems se encuentran diferencias estadísticamente significativas, el grupo de control presenta una mejor puntuación media en el MEC y el grupo experimental tiene una media mayor de años de escolarización (10.3 vs. $8.8 ; U=764.0 ; p<.05)$.

\section{Instrumentos}

Para evaluar el estado cognitivo general se empleó el Mini-Examen Cognoscitivo General (MEC-35) (Lobo, Ezquerra, Gómez, Sala, \& Seva, 1979) con punto de corte 23.

Para la evaluación de la FVS se utilizaron tres categorías semánticas: "animales" (Carnero, 1999; Carnero, et al., 2000; Guardia, et al., 1997; Peña-Casanova, Guardia, BertránSerra, Manero, \& Jarne, 1997; Spreen \& Strauss, 1998), "cosas en una casa" (Fernández, et al., 2002), y "alimentos" (Moreno, Adrián, Buiza, \& González, 2004). Para su aplicación se empleó la propuesta de conceder 60 segundos para cada categoría, anotando los aciertos cada 15 segundos (Ramírez, et al., 2005). La consigna para las diferentes categorías fue textualmente: "Le voy a pedir que me diga todos... (los nombres de animales/cosas que hay en una casa/los nombres de alimentos) que se le ocurran, tiene un minuto; yo le voy a decir cuándo pare de hacerlo".

Para la evaluación de la FVF se emplearon los fonemas "F", "A", "S", "P", "M" y "R". Las instrucciones dadas fue- 
ron las descritas por Spreen y Strauss (Spreen \& Strauss, 1998) y se registró la cantidad de palabras correctas que el sujeto verbalizó durante un minuto para cada fonema, anotando los aciertos cada 15 segundos.

En la puntuación no se consideraron las perseveraciones (palabras repetidas en el mismo criterio de evocación), las intrusiones (palabras que no pertenecían a la categoría explorada o palabras que no empezaban por la letra requerida), el cambio de género ("rico"-"rica"), los nombres propios de animales, las variaciones de denominación intraespecie ("caballo"-"yegua", "caballo"- "potro"), las razas de un tipo de animal ("pastor alemán") ni categorías supraordenadas ("pez", "pájaro", "insecto"). Se aceptaron el nombre, el verbo y el adjetivo de una misma raíz ("asador", "asar" y "asado"). No se proporcionaron ejemplos ni se ofrecieron ayudas durante el tiempo de la prueba, pero si la persona se detenía antes de completar el minuto, se le motivaba a seguir. No se comunicó a los sujetos los criterios de calificación.

\section{Procedimiento}

Previamente a la aplicación del protocolo de evaluación se entregó a los sujetos un consentimiento informado, indicando la aceptación voluntaria de su participación en la investigación. Seguidamente se administró el miniexamen cognitivo MEC-35 (Lobo, et al., 1979) con el fin de excluir de la muestra del grupo control a personas con deterioro cognitivo. Después se pasaron, en este orden, las pruebas de FVS "animales", "cosas en una casa" y "alimentos" y de FVF con los fonemas "F", "A", "S" "P", "M" y "R". Cada entrevista duró entre 20-25 minutos.

\section{Análisis de datos}

Se realizaron análisis descriptivos para todas las variables. En los análisis bivariados entre los sujetos de los grupos control y experimental, se empleó el análisis de $\chi^{2}$, o la prueba no paramétrica " $U$ " de Mann-Withney, según la naturaleza de las variables analizadas, considerándose una $p<.05$ como significativa. Además se valoraron dentro de cada grupo la ejecución en el primer periodo (0-30") y en el segundo (31"-60"). Para el contraste estadístico se utilizó la prueba $t$ para muestras relacionadas. Posteriormente se realizaron análisis de correlaciones no paramétricas entre las pruebas de fluencia verbal semántica y fonológica.

Para el análisis multivariado y el establecimiento de puntos de corte entre los grupos experimental y de control se empleó el análisis de segmentación CHAID (Chi-squared $A u$ tomatic Interaction Detection). Esta técnica, desarrollada por diferentes autores (Bouroche \& Tennenhaus, 1972; Cellard, Labbe, \& Cox, 1967; Kass, 1980; Madgison, 1989), valora la capacidad discriminante de una variable nominal (en este caso a la asignación al grupo de control o experimental) mediante la significación de la $X^{2}$ y ha sido empleada con bue- nos resultados tanto en población normal (Peñalva \& LópezGoñi, 2014) como en clínica (López-Goñi, FernándezMontalvo, \& Arteaga, 2012). El análisis se desarrolló en cuatro pasos sucesivos En un primer momento se valoraron las pruebas de FVS de forma individual y conjunta. En un segundo paso se valoraron del mismo modo las pruebas de FVF para posteriormente valorar conjuntamente la capacidad predictiva de las pruebas de FVS y de FVF. Finalmente se incorporaron las variables sociodemográficas junto con las pruebas de FV.

Todos los análisis estadísticos se realizaron con el paquete estadístico SPSS (vs. 15.0).

\section{Resultados}

\section{Pruebas de FVS y FVF}

En la Tabla 1 se presenta la ejecución de los sujetos en las pruebas de FV. La prueba de FVS más productiva en el caso del grupo de control es "cosas que hay en una casa" con una media de 17.2 ítems $(D T=5.5)$ seguida por "alimentos" $(M=15.7 ; D T=4.9)$ y "animales" $(M=13.9 ; D T$ $=4.4)$. La diferencia media de producción entre la prueba mayor y menor es de 3.3 ítems.

En el grupo experimental la diferencia de producción total no llega a un ítem entre las tres pruebas: "alimentos" $(M$ $=10.4 ; D T=4.0)$, "cosas que hay en una casa" $(M=10.1$; $D T=4.9)$ y "animales" $(M=9.6 ; D T=3.6)$. En todos los casos, los sujetos del grupo de control producen más ítems que los pacientes del experimental.

Por lo que respecta a las pruebas de FVF (Tabla 1), la producción total en cada letra sigue el mismo orden en los dos grupos, siendo la "P" la más productiva, seguida por "R", "M", "S", “A" y "F". En todos los casos la producción del grupo de control es mayor que la del experimental, y la diferencia entre las letras más ("P") y menos productiva ("F") es de 2.8 ítems para el grupo de control y de 2.4 para el experimental.

En cuanto a la ejecución intragrupo en los periodos 030 " y 31 " $-60 "$ tanto en el grupo control como en el experimental la ejecución en todas las tareas fue significativamente superior en el primer periodo respecto al segundo.

\section{Correlación entre las pruebas de FVS - FVF}

En la Tabla 2 se presentan las correlaciones entre las pruebas de FVS y FVF.

La correlaciones entre las pruebas de FVS se hallan entre .72 (“alimentos"-“animales") y .77 (“animales"-“cosas") y entre las de FVF entre .64 ("S"-“F”) y .78 (para "M"--P" y "R"-“M"). A su vez, las correlaciones entre las pruebas de FVS y FVF oscilan entre .38 ("R"-_animales") y .57 ("M""alimentos"). 
Tabla 1. Descriptivos pruebas fluidez verbal semántica y fonológica (experimental vs. control).

\begin{tabular}{|c|c|c|c|c|c|c|c|c|c|}
\hline & & \multicolumn{2}{|c|}{ Total } & \multicolumn{2}{|c|}{ Experimental } & \multicolumn{2}{|c|}{ Control } & \multirow[b]{2}{*}{$U$} & \multirow{2}{*}{$\begin{array}{c}\text { Dif. } \\
\text { (C-E) }\end{array}$} \\
\hline & & $M$ & $(D T)$ & $M$ & (DT) & $M$ & (DT) & & \\
\hline \multirow{3}{*}{ Animales } & $0-60^{\prime \prime}$ & 11.7 & $(4.6)$ & 9.6 & $(3.6)$ & 13.9 & $(4.4)$ & $527.5^{* * *}$ & 4.4 \\
\hline & $0-30^{\prime \prime}$ & 8.2 & $(3.0)$ & 6.8 & $(2.5)$ & 9.6 & $(2.7)$ & $533.0 * * *$ & 2.8 \\
\hline & $31^{\prime \prime}-60^{\prime \prime}$ & 3.7 & $(2.7)$ & 2.8 & $(2.0)$ & 4.7 & $(3.0)$ & $724.0 * * *$ & 1.9 \\
\hline \multirow{3}{*}{ Cosas } & $0-60^{\prime \prime}$ & 13.6 & $(6.3)$ & 10.1 & $(4.9)$ & 17.2 & $(5.5)$ & $405.0^{* * *}$ & 7.1 \\
\hline & $0-30^{\prime \prime}$ & 8.3 & $(3.9)$ & 6.2 & $(3.3)$ & 10.5 & $(3.3)$ & $423.5^{* * *}$ & 4.3 \\
\hline & $31^{\prime \prime}-60 "$ & 5.6 & $(3.7)$ & 3.9 & $(2.7)$ & 7.4 & $(3.7)$ & $500.0^{* * *}$ & 3.5 \\
\hline \multirow{3}{*}{ Alimentos } & $0-60^{\prime \prime}$ & 13.0 & $(5.2)$ & 10.4 & $(4.0)$ & 15.7 & $(4.9)$ & $488.5^{* * *}$ & 5.3 \\
\hline & $0-30^{\prime \prime}$ & 8.0 & $(3.2)$ & 6.3 & $(2.5)$ & 9.7 & $(2.9)$ & $460.0^{* * *}$ & 3.4 \\
\hline & $31 "-60 "$ & 5.2 & $(2.9)$ & 4.1 & $(2.3)$ & 6.4 & $(3.0)$ & $666.0^{* * *}$ & 2.3 \\
\hline \multirow{3}{*}{$\mathrm{F}$} & $0-60^{\prime \prime}$ & 6.6 & $(3.8)$ & 5.3 & $(3.4)$ & 8.0 & $(3.7)$ & $730.0^{* * *}$ & 2.8 \\
\hline & $0-30^{\prime \prime}$ & 4.1 & $(2.7)$ & 3.1 & $(2.3)$ & 5.1 & $(2.6)$ & $726.5^{* * *}$ & 2 \\
\hline & $31^{\prime \prime}-60^{\prime \prime}$ & 2.6 & $(1.9)$ & 2.2 & $(1.6)$ & 2.9 & $(2.0)$ & 982.5 & 0.7 \\
\hline \multirow{3}{*}{ A } & $0-60^{\prime \prime}$ & 7.2 & $(3.6)$ & 5.5 & $(3.0)$ & 8.8 & $(3.5)$ & $596.5^{* * *}$ & 3.3 \\
\hline & $0-30^{\prime \prime}$ & 4.6 & $(2.4)$ & 3.6 & $(2.0)$ & 5.6 & $(2.4)$ & $623.0^{* * *}$ & 2 \\
\hline & $31^{\prime \prime}-60 "$ & 2.5 & $(1.7)$ & 1.9 & $(1.4)$ & 3.1 & $(1.6)$ & $720.0^{* * *}$ & 1.2 \\
\hline \multirow{3}{*}{ S } & $0-60^{\prime \prime}$ & 7.3 & $(3.8)$ & 5.7 & $(3.1)$ & 8.9 & $(3.8)$ & $650.0^{* * *}$ & 3.2 \\
\hline & $0-30^{\prime \prime}$ & 4.8 & $(2.5)$ & 3.8 & $(2.1)$ & 5.8 & $(2.4)$ & $667.5^{* * *}$ & 2 \\
\hline & $31^{\prime \prime}-60^{\prime \prime}$ & 2.5 & $(1.9)$ & 1.9 & $(1.5)$ & 3.2 & $(2.1)$ & $806.5^{* *}$ & 1.3 \\
\hline \multirow[t]{2}{*}{ Total FAS } & & 21.0 & $(9.8)$ & 16.5 & $(8.1)$ & 25.5 & $(9.4)$ & $582.0^{* * *}$ & 9.0 \\
\hline & $0-60^{\prime \prime}$ & 9.3 & $(3.9)$ & 7.7 & $(3.2)$ & 10.8 & $(4.0)$ & $681.0^{* * *}$ & 3.1 \\
\hline \multirow[t]{3}{*}{$\mathrm{P}$} & $0-30^{\prime \prime}$ & 5.9 & $(2.4)$ & 4.9 & $(2.1)$ & 6.8 & $(2.2)$ & $653.5^{* * *}$ & 1.9 \\
\hline & $31^{\prime \prime}-60^{\prime \prime}$ & 3.4 & $(2.2)$ & 2.8 & $(1.6)$ & 4.0 & $(2.5)$ & $916.0 *$ & 1.2 \\
\hline & $0-60^{\prime \prime}$ & 7.9 & $(3.6)$ & 6.5 & $(3.0)$ & 9.3 & $(3.5)$ & $705.0^{* * *}$ & 2.8 \\
\hline \multirow[t]{3}{*}{ M } & $0-30^{\prime \prime}$ & 4.9 & $(2.3)$ & 4.1 & $(2.1)$ & 5.7 & $(2.2)$ & $737.5^{* * *}$ & 1.6 \\
\hline & $31^{\prime \prime}-60 " \prime$ & 2.9 & $(1.9)$ & 2.3 & $(1.4)$ & 3.5 & $(2.0)$ & $811.0^{* *}$ & 1.2 \\
\hline & $0-60^{\prime \prime}$ & 8.5 & $(3.7)$ & 7.3 & $(3.7)$ & 9.7 & $(3.4)$ & $776.5^{* *}$ & 2.4 \\
\hline \multirow[t]{2}{*}{$\mathrm{R}$} & $0-30^{\prime \prime}$ & 5.6 & $(2.6)$ & 4.5 & $(2.4)$ & 6.6 & $(2.2)$ & $621.0 * * *$ & 2.1 \\
\hline & $31^{\prime \prime}-60 "$ & 2.9 & $(1.8)$ & 2.8 & $(1.7)$ & 3.1 & $(1.8)$ & 1122.0 & 0.3 \\
\hline Total PMR & & 25.6 & $(10.3)$ & 21.4 & $(8.8)$ & 29.8 & $(9.9)$ & $651.5^{* * *}$ & 8.5 \\
\hline
\end{tabular}

${ }^{*} p<.05 ; * * p<.01 ; * * * p<.001$

Tabla 2. Correlaciones entre pruebas de fluidez verbal semántica y fluidez fonológica.

\begin{tabular}{|c|c|c|c|c|c|c|c|c|c|c|}
\hline & Cosas & Alimentos & $\mathrm{F}$ & A & $\mathrm{S}$ & FAS & $\mathrm{P}$ & $\mathrm{M}$ & $\mathrm{R}$ & PMR \\
\hline Animales & $.77^{*}$ & $.72^{*}$ & $.43^{*}$ & $.40^{*}$ & $.40^{*}$ & $.46^{*}$ & $.54^{*}$ & $.51 *$ & $.38^{*}$ & $.52 *$ \\
\hline Cosas & 1 & $.75^{*}$ & $.41 *$ & $.45^{*}$ & $.40^{*}$ & $.48^{*}$ & $.56^{*}$ & $.52 *$ & $.43^{*}$ & $.56^{*}$ \\
\hline Alimentos & & 1 & $.45^{*}$ & $.48^{*}$ & $.44^{*}$ & $.53 *$ & $.55^{*}$ & $.57^{*}$ & $.46^{*}$ & $.59 *$ \\
\hline $\mathrm{F}$ & & & 1 & $.67^{*}$ & $.64 *$ & $.87^{*}$ & $.69 *$ & $.66^{*}$ & $.67^{*}$ & $.74 *$ \\
\hline A & & & & 1 & $.69^{*}$ & $.88^{*}$ & $.67 *$ & $.64^{*}$ & $.68^{*}$ & $.74 *$ \\
\hline S & & & & & 1 & $.86^{*}$ & $.72 *$ & $.71 *$ & $.65^{*}$ & $.77 *$ \\
\hline FAS & & & & & & 1 & $.78^{*}$ & $.75^{*}$ & $.75^{*}$ & $.85^{*}$ \\
\hline $\mathrm{P}$ & & & & & & & 1 & $.78^{*}$ & $.70^{*}$ & $.90 *$ \\
\hline M & & & & & & & & 1 & $.78^{*}$ & $.92 *$ \\
\hline $\mathrm{R}$ & & & & & & & & & 1 & $.90 *$ \\
\hline
\end{tabular}

\section{Valor predictivo de las pruebas de FVS - FVF}

En la Tabla 3 se presentan los diferentes puntos de corte y la capacidad predictiva para cada prueba de FV, así como la combinación de las tareas de FVS y de FVF.

Las pruebas de FVS consiguen clasificar correctamente entre el 75 y $79 \%$ de la muestra, consiguiendo al combinar las pruebas de "cosas que hay en una casa" y "animales" clasificar correctamente al 82\% de los sujetos.

Las tareas fonológicas consiguen clasificar correctamente entre el 65 y 74\% de los sujetos y la combinación entre ellas no llega a mejorar la clasificación de los fonemas "A" y "M" considerados individualmente.

\section{Valor predictivo conjunto de las pruebas de FVS - FVF}

En la Figura 1 se presenta el árbol resultante del procedimiento CHAID realizado. Con este árbol se predice correctamente la asignación del $83 \%$ de la muestra (86\% del grupo experimental y $80 \%$ del grupo de control). 
Tabla 3. Puntos de corte para las pruebas de FVS y FVF.

\begin{tabular}{|c|c|c|c|c|c|c|c|c|c|c|}
\hline & \multirow{3}{*}{ Modelo } & \multirow{3}{*}{$\begin{array}{c}\text { Variables } \\
\text { incorporadas }\end{array}$} & \multirow{3}{*}{$\begin{array}{l}\text { Ptos. } \\
\text { Corte }\end{array}$} & \multicolumn{6}{|c|}{ Clasificaciones correctas } & \multirow{3}{*}{$X^{2}$} \\
\hline & & & & \multicolumn{2}{|c|}{$\begin{array}{c}\text { Total } \\
(N=100)\end{array}$} & \multicolumn{2}{|c|}{$\begin{array}{c}\text { Experimental } \\
(n=50)\end{array}$} & \multicolumn{2}{|c|}{$\begin{array}{l}\text { Control } \\
(n=50)\end{array}$} & \\
\hline & & & & $\mathrm{n}$ & $(\%)$ & $\mathrm{n}$ & $(\%)$ & $\mathrm{n}$ & $(\%)$ & \\
\hline & 1 & Animales & 13 & 77 & $(77 \%)$ & 43 & $(86 \%)$ & 34 & $(68 \%)$ & $30.1 * *$ \\
\hline & 2 & Cosas & 16 & 79 & $(79 \%)$ & 45 & $(90 \%)$ & 34 & $(68 \%)$ & $35.3^{* *}$ \\
\hline & 3 & Alimentos & 9 y 14 & 75 & $(75 \%)$ & 41 & $(82 \%)$ & 34 & $(68 \%)$ & $29.0 * *$ \\
\hline FVS & 4 Animales-Cosas-Alimentos & $\begin{array}{c}\text { Cosas } \\
\text { Animales }\end{array}$ & $\begin{array}{l}16 \\
13 \\
\end{array}$ & 82 & $(82 \%)$ & 41 & $(82 \%)$ & 41 & $(82 \%)$ & $\begin{array}{c}35.3^{* *} \\
9.7^{*}\end{array}$ \\
\hline \multirow{9}{*}{ FVF } & 5 & "F" & 4 & 65 & $(65 \%)$ & 20 & $(40 \%)$ & 45 & $(90 \%)$ & $12.0^{* *}$ \\
\hline & 6 & "A" & 8 & 71 & $(71 \%)$ & 39 & $(78 \%)$ & 32 & $(64 \%)$ & $18.0^{* *}$ \\
\hline & 7 & "S" & 2 y 9 & 69 & $(69 \%)$ & 43 & $(86 \%)$ & 26 & $(52 \%)$ & $20.1 * *$ \\
\hline & 8 "F"-“A"-“S" & "A" & 8 & 71 & $(71 \%)$ & 39 & $(78 \%)$ & 32 & $(64 \%)$ & $18.0^{* *}$ \\
\hline & $\overline{9}$ & "P" & 5 y 14 & 64 & $(64 \%)$ & 48 & $(96 \%)$ & 16 & $(32 \%)$ & $17.4^{*}$ \\
\hline & 10 & "M" & 7,10 y 11 & 74 & $(74 \%)$ & 40 & $(80 \%)$ & 34 & $(68 \%)$ & $25.5^{*}$ \\
\hline & 11 & "R" & 9 & 67 & $(67 \%)$ & 32 & $(64 \%)$ & 35 & $(70 \%)$ & $11.6^{*}$ \\
\hline & 12 P"-M"-“R” & "M" & 7,10 y 11 & 74 & $(74 \%)$ & 40 & $(80 \%)$ & 34 & $(68 \%)$ & $25.5^{*}$ \\
\hline & 13 “F”-“A”-“S”-“"P”-M"-_R” & "A" & 8 & 71 & $(71 \%)$ & 39 & $(78 \%)$ & 32 & $(64 \%)$ & $18.0^{* *}$ \\
\hline
\end{tabular}

FVS = Fluencia Verbal Semántica; FVF = Fluencia Verbal Fonológica.

${ }^{*} p<.01 ; * * p<.001$.

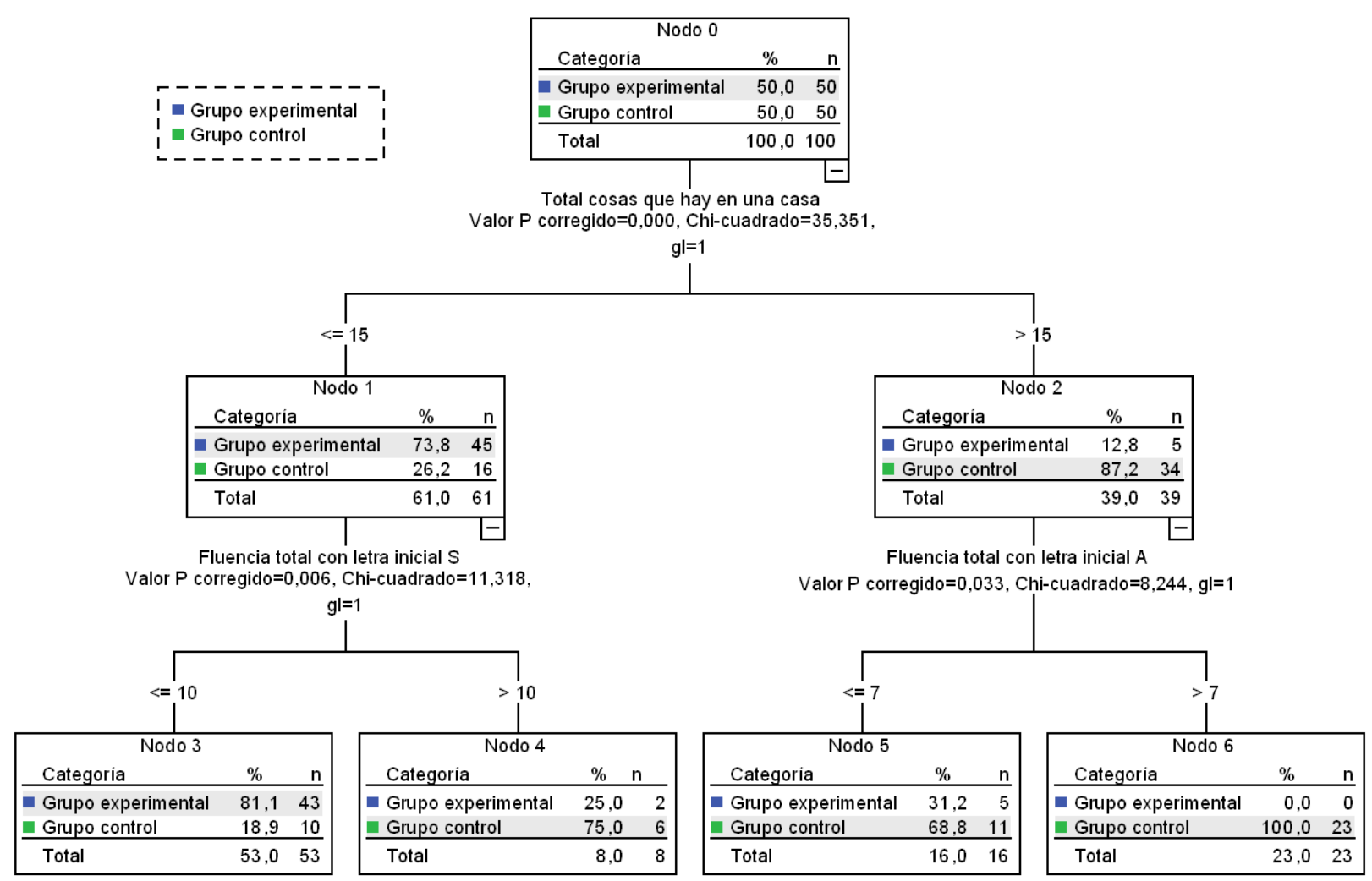

\begin{tabular}{lccc}
\multicolumn{4}{c}{ Clasificación } \\
\cline { 2 - 4 } Observado & Pronosticado & \\
\cline { 2 - 4 } & Grupo experimental & Grupo control & Porcentaje correcto \\
\hline Grupo experimental & 43 & 7 & $86.0 \%$ \\
\hline Grupo control & 10 & 40 & $80.0 \%$ \\
\hline Porcentaje global & $53.0 \%$ & $47.0 \%$ & $83.0 \%$ \\
\hline
\end{tabular}

Figura 1. Árbol CHAID y bondad de ajuste para las pruebas de FV. 
De las tres pruebas de FVS, el modelo seleccionó como mejor predictor el punto de corte de 15 para la tarea "cosas que hay en una casa". De las 39 personas que obtienen más de 15 ítems en esta prueba, el 87.2\% $(n=34)$ pertenecen al grupo de control. Quienes además producen más de 7 ítems en la prueba de FVF con el fonema "A" $(n=23)$ todas pertenecen al grupo de control.

Por otra parte, entre quienes han obtenido menos de 16 ítems en la tarea "cosas que hay en una casa" tan sólo dos sujetos del grupo experimental obtenían más de 10 ítems en la prueba de FVF con el fonema "S".

\section{Valor predictivo de las pruebas de FVS-FVF junto con las variables sociodemográficas}

Cuando en el análisis CHAID se incorporan las variables sociodemográficas, el modelo selecciona la edad y la variable años de escolarización (Figura 2), para clasificar correctamente al $91 \%$ de los sujetos evaluados (el 96\% de los pacientes con EA y el $86 \%$ de los sujetos del grupo de control).

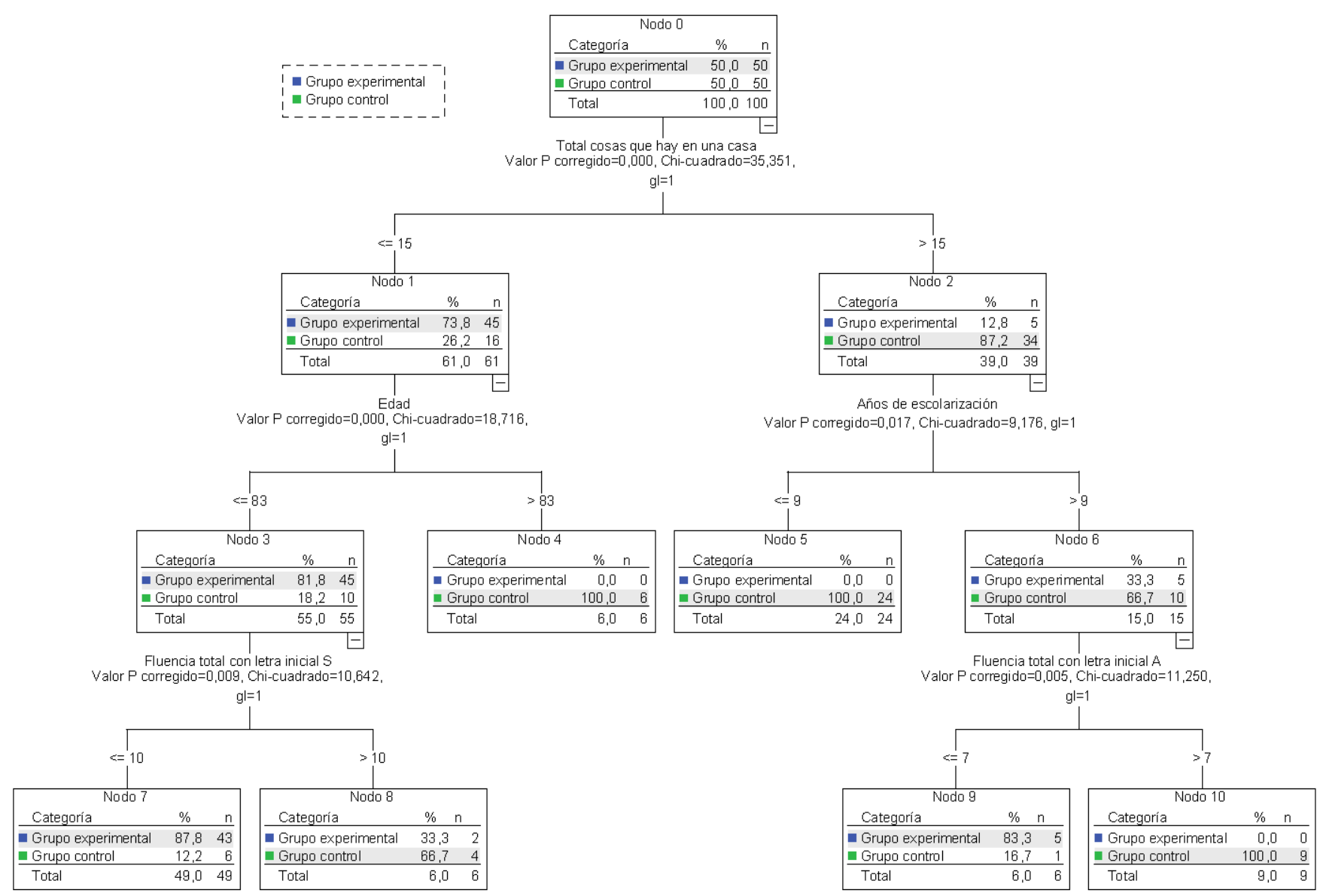

Clasificación

\begin{tabular}{|c|c|c|c|}
\hline \multirow[t]{2}{*}{ Observado } & \multicolumn{3}{|c|}{ Pronosticado } \\
\hline & $\begin{array}{c}\text { Grupo } \\
\text { experimental }\end{array}$ & $\begin{array}{l}\text { Grupo } \\
\text { control }\end{array}$ & $\begin{array}{c}\text { Porcentaje } \\
\text { correcto }\end{array}$ \\
\hline Grupo experimental & 48 & 2 & $96.0 \%$ \\
\hline Grupo control & 7 & 43 & $86.0 \%$ \\
\hline Porcentaje global & $55.0 \%$ & $45.0 \%$ & $91.0 \%$ \\
\hline
\end{tabular}

Figura 2. Árbol CHAID y bondad de ajuste para las pruebas de FV y sociodemográficas.

Para ello el modelo mantiene el punto de corte de 15 para "cosas que hay en una casa, pero para los 39 sujetos que producen más de 15 respuestas incorpora la variable años de escolarización. Por debajo de 9 años de escolarización todos 
los sujetos $(n=24)$ pertenecen al grupo de control. Además, entre los 15 sujetos con más de 9 años, todos aquellos que producen más de 7 ítems con el fonema "A" $(n=9)$ pertenecen al grupo de control.

Por debajo de 15 puntos en la tarea "cosas que hay en una casa", el modelo incorpora la edad. Todos los mayores de 83 años $(n=6)$ pertenecían al grupo de control. Entre el resto de sujetos $(n=55)$ se incorpora nuevamente las producción en el fonema "S" pero con el punto de corte 10. El $87.8 \%(n=43)$ de quienes obtienen esta puntuación o menor pertenecen al grupo de control.

\section{Discusión}

Este estudio muestra la utilidad clínica del empleo de diferentes variables sociodemográficas, semánticas, y fonológicas, para discriminar entre un grupo de pacientes con EA y un grupo de control. La mejor valoración de EA se hace con la combinación de "cosas que hay en una casa" y los fonemas "A" y "S" junto con las variables edad y años de escolarización. No obstante, las tres categorías semánticas empleadas ("cosas en una casa", "animales" y "alimentos") y los diferentes fonemas ("F"-“A"-“S"-"P"-“M"-"R") muestran unos resultados muy semejantes entre sí y mantienen la validez de criterio en la exploración neuropsicológica.

De hecho, se ha obtenido una alta correlación entre las tres tareas de FVS (con correlaciones superiores a .71) lo que indica que la categoría "alimentos" sirve como forma paralela de exploración a las categorías ampliamente utilizadas de “animales", "cosas en una casa" (Fernández, et al., 2002) y la tarea del "supermercado" (Garcés-Redondo, et al., 2004).

Para el grupo con EA no hay diferencia de producción entre las tres categorías semánticas, lo que nuevamente avala la intercambiabilidad entre estas pruebas. Ahora bien, para el grupo de control la categoría de "cosas en una casa" es la más productiva, lo que podría explicarse por una mayor familiaridad con dicha categoría y/o por un menor grado de dificultad (Pascual, et al., 2000).

Para "animales" los resultados de este trabajo se colocan en una posición intermedia con una emisión de 14 palabras en población sana, frente a 11 y 16 de otras investigaciones (Carnero-Pardo \& Lendínez-González, 1999; Salorio, Navarro, Navarro, \& López, 2008; Tombaugh, et al., 1999). Los pacientes con demencia producen como media 9.6 ítems frente a 10 y 6 en los mismos estudios. El resultado de esta investigación también es semejante al del grupo Del Ser en su estandarización de una amplia batería de pruebas neuropsicológicas desde una perspectiva poblacional (Del Ser, et al., 2004).

Las tareas de FVF producen menor número de palabras que la FVS para ambos grupos y, en ambos casos, la mayor producción de palabras se produce en los 30 primeros segundos de la prueba. Estos resultados son concordantes con los encontrados en población adulta castellano parlante $\mathrm{Bu}-$ riel, Fombuena, Böhm, Rodés, \& Peña-Casanova, 2004; Butman, Allegri, Harris, \& Drake, 2000; Fernández-Turrado, et al., 2007), lo que hace pensar en una mayor complejidad de la tarea fonológica frente a la semántica (Alberca, et al., 1999; Marino \& Alderete, 2010; Villodre, et al., 2006). Resultado interesante, ya que la demanda cognitiva puede no ser uniforme a lo largo del desarrollo de las pruebas de FV y, el grado de esfuerzo cognitivo sea mayor durante la segunda mitad (30-60 seg) respecto a la primera. Este mayor esfuerzo puede derivar de una mayor demanda de atención sostenida, de memoria de trabajo y de búsqueda lexical en la memoria semántica, entre otros (Pascual, Fernández, Santos, Iñíguez, Ríos, et al., 2001; Pascual, Fernández, Santos, Iñíguez, Tejero, et al., 2001). También se relaciona con el modelo de reserva cognitiva, que implica la activación progresiva de redes neuronales en respuesta a demandas crecientes o con la activación de redes adicionales para maximizar el rendimiento (Carnero-Pardo \& Del Ser, 2007; Stern, 2002). Por ello parece lógica la estandarización de cada una de las mitades de las tareas como un instrumento adicional para interpretar el rendimiento en dos partes simétricas en el tiempo pero con distinto grado de dificultad (Fernández-Turrado, et al., 2007).

En cuanto a los dos grupos de fonemas empleados, para "F"-"A"-"S" se obtiene un resultado casi idéntico, al presentado por Rami (Rami, Serradell, Bosch, Villar, \& Molinuevo, 2007) estratificado por nivel de escolaridad.

En este estudio la fluencia de "P"-"M"-"R" es mayor que la de "F"-“A"-"S" para ambos grupos, aunque sin diferencias estadísticamente significativas. Las correlaciones más altas entre fonemas se dan entre "P" y "S" $(r=.72)$ y entre "M" $\mathrm{y}$ " $\mathrm{S}$ " $(r=.71)$. Es interesante destacar que entre las series de fonemas recomendados no se encuentran combinaciones de "P" y "S" o de "M" y "S" cuando en este estudio han obtenido las correlaciones más altas. De hecho, la mayoría de los estudios que optan por el uso de tríadas de fonemas se decantan por la tríada "F" - "A"- "S".

Por otro lado, se ha hallado una correlación moderada (con valores comprendidos entre .38 y .57) entre las tareas semánticas y fonológicas. Este dato corroboraría la existencia de procesos cognitivos y regiones cerebrales diferentes, la semántica se relaciona con la activación de áreas frontales y temporales bilaterales y la fonológica con áreas frontales. Al considerarlas conjuntamente mejoran la capacidad discriminante de las pruebas tal y como se ha encontrado en los modelos multivariantes presentados. Así, los últimos hallazgos indican que la discrepancia entre ambas fluencias como algo característico de la EA en la que la fluencia semántica está más dañada que la fonémica y declina más rápido (Clark, et al., 2009; Henry, Crawford, \& Phillips, 2004) podría tratarse de una tendencia que se ha exagerado (Laws, Duncan, \& Gale, 2010).

La combinación de las pruebas de FVS y FVF mejora la discriminación entre población sana y con demencia. En los dos modelos finales desarrollados (ilustraciones 1 y 2) la FVS establece un primer punto de corte, para posteriormente incorporar las pruebas de FVF (con "A" y "S" que sí son los que habitualmente se incorporan en las tareas con tría- 
das). Parece que las pruebas de FVF aportasen una discriminación más fina respecto a otra más grosera, la semántica. Cuando se incorporan en el modelo las variables edad y años de escolarización éstas se sitúan entre ambos tipos de fluencia, mediando entre los valores extremos. Estos resultados son coherentes con otros informados previamente (Butman, et al., 2000; Gonzalez da Silva, Magnus Petersson, Faísca, Ingvar, \& Reis, 2004; Marino \& Alderete, 2010; Rami, et al., 2007; Salorio, et al., 2008; Tombaugh, et al., 1999) y muestran el posible efecto de la escolarización sobre el rendimiento en las pruebas de FV.

En este estudio la variable sexo no ha sido incluida por el modelo, lo cual podría indicar que, efectivamente, las variables relevantes son las relacionadas con el nivel educativo y que estudios anteriores recogían no tanto el efecto de una variable sexo como la menor escolarización de las mujeres (Carnero, 1999).

Este estudio presenta, no obstante, algunas limitaciones por lo que hay que ser cauto a la hora de interpretar los resultados. En primer lugar sería deseable replicar los datos de esta investigación con otras muestras clínicas de pacientes,

\section{Referencias}

Alamo, C. D., Mir, M. L., Olivares, T., Barroso, J., \& Nieto, A. (1999). Efecto de la edad, nivel educativo y estado cognitivo general sobre la fluidez verbal en hispanobablantes. Datos normativos preliminares. Paper presented at the First International Congress on Neuropsychologiy in internet. http://www.uninet.edu/union99/congress/libs/val/v01.html

Alberca, R., Salas, D., Pérez-Gil, J. A., Lozano, P., \& Gil-Néciga, E. (1999). Verbal fluency and Alzheimer's disease. Neurología, 14, 344-348.

Artiola, L., Hermosillo, D., Heaton, R., \& Pardee, R. E. (1999). Manual de normas y procedimientos para la batería neuropsicológica en español: Tucson, Az.

Barry, D., Bates, M. E., \& Labouvie, E. (2008). FAS and CFL Forms of verbal fluency differ in difficulty: a meta-analytic study. Applied neuropsychology, 15(2), 97-106.

Bouroche, J. M., \& Tennenhaus, M. (1972). Some segmentation methods. Metra, 7, 407-418.

Buriel, Y., Fombuena, N. G., Böhm, P., Rodés, E., \& Peña-Casanova, J. (2004). Fluencia verbal. Estudio normativo piloto en una muestra Española de adultos jóvenes (20 a 49 años). Neurologia, 19(4), 153-159.

Butman, J., Allegri, R. F., Harris, P., \& Drake, M. (2000). Fluencia verbal en Español: Datos normativos en Argentina. Medicina, 60(5), 561-564.

Canning, S. J. D., Leach, L., Stuss, D., Ngo, L., \& Black, S. E. (2004). Diagnostic utility of abbreviated fluency measures in Alzheimer disease and vascular dementia. Neurology, 62(4), 556-562.

Carnero-Pardo, C. (2000). Educación, demencia y reserva cerebral. Revista de Neurologia, 31(6), 584-592.

Carnero-Pardo, C., \& Del Ser, T. (2007). La educación proporciona reserva cognitiva en el deterioro cognitivo y la demencia. Neurología, 22(2), 7885 .

Carnero-Pardo, C., \& Lendínez-González, A. (1999). Utilidad del test de fluencia verbal semantica en el diagnostico de demencia. Revista de neurología, 29(8), 709-714.

Carnero, C. (1999). Fluencia verbal semántica en pacientes neurológicos sin demencia y bajo nivel educativo. Revista de neurología, 28(9), 858-862.

Carnero, C., Maestre, J., Marta, J., Mola, S., Olivares, J., \& Sempere, A. P. (2000). Validación de un modelo de predicción de fluidez verbal semántica. Revista de neurología, 30(11), 1012-1015.

Cellard, J. C., Labbe, B., \& Cox, G. (1967). Le programme Elisée. Presentation et application. Metra, 3, 511-519.

Clark, L. J., Gatz, M., Zheng, L., Chen, Y. L., McCleary, C., \& Mack, W. J. (2009). Longitudinal verbal fluency in normal aging, preclinical, and puesto que el nivel de afectación cognitiva podría ser diferente. Asimismo, se podría variar el orden de pasación de las pruebas para controlar el efecto de la fatiga que pudiera estar afectando a los resultados. En último lugar es recomendable continuar con la labor de normativización de estas pruebas para mejorar su eficacia diagnóstica.

\section{Conclusiones}

El objetivo de este trabajo ha sido proponer tareas alternativas a ambas categorías de FV tanto para contribuir a diferenciar población con EA frente a sujetos sanos, como para disponer de otras pruebas en las exploraciones neuropsicológicas repetidas. Se propone un modelo predictivo que, con un uso combinado de ambas tareas y teniendo en cuenta variables sociodemográficas, con la exploración de "cosas en una casa" y los fonemas "A" y "S", con un mínimo de tiempo para su aplicación, se logra discriminar al 91\% de los sujetos evaluados.

prevalent Alzheimer's disease. American Journal of Alzheimer's disease and other dementia, 24(6), 461-468.

Contador, I., Fernández-Calvo, B., Ramos, F., Tapias-Merino, E., \& Bermejo-Pareja, F. (2010). El cribado de la demencia en atención primaria. Revisión crítica. Revista de neurologia, 51(11), 677-686.

Cuetos-Vega, F., Menéndez-González, M., \& Calatayud-Noguera, T. (2007) Descripción de un nuevo test para la detección precoz de la enfermedad de Alzheimer. Revista de neurología, 44(8), 469-474.

Del Ser, T., García, M. J., Sánchez, F., Frades, B., Rodríguez, A., Bartolomé, M. P., et al. (2004). Evaluación cognitiva del anciano. Datos normativos de una muestra poblacional española de más de 70 años Medicina Clínica, 122(19), 727-740.

Fernández-Turrado, T., Pascual-Millán, L. F., Fernández-Arín, E., LarrodéPellicer, P., Santos-Lasaosa, S., \& Mostacero-Miguel, E. (2007). Modelo de análisis en dos mitades para tareas de fluidez semántica. Revista de neurología, 44(9), 531-536.

Fernández, T., Ríos, C., Santos, S., Casadevall, T., Tejero, C., López-García E., et al. (2002). Cosas en una casa, una tarea alternativa a 'animales' en la exploración de la fluidez verbal semántica: estudio de validación. Revista de neurología, 35(6), 520-523.

Galeote, M. A., \& Peraita, H. (1999). Memoria semántica y fluidez verbal en demencias Revista española de neuropsicología, 1(2-3), 3-18.

Garcés-Redondo, M., Santos, S., Pérez-Lázaro, C., \& Pascual-Millán, F. (2004). Test del supermercado: datos normativos preliminares en nuestro medio. Revista de neurología, 39(5), 415-418.

Gonzalez da Silva, C., Magnus Petersson, K., Faísca, L., Ingvar, M., \& Reis, A. (2004). The Effects of Literacy and Education on the Quantitative and Qualitative Aspects of Semantic Verbal Fluency. Journal of Clinical and Experimental Neuropsychology, 26(2), 266-277.

Guardia, J., Peña-Casanova, J., Bertrán-Serra, I., Manero, R. M., Meza, M., \& Böhm, P. (1997). Versión abreviada del test Barcelona (II): puntuación global normalizada. Neurología 1997; 12: 112-6. Neurología, 12, 112-116.

Henry, J. D., Crawford, J. R., \& Phillips, L. H. (2004). Verbal fluency performance in dementia of the Alzheimer's type: a meta-analysis. Neuropsychologia, 42(9), 1212-1222.

Kass, G. (1980). An exploratory technique for investigating large quantities of categorical data. Applied Statistics 29, 119-127.

Kertesz, A., \& Mohs, R. C. (1996). Cognition. In S. Gauthier (Ed.), Clinical diagnosis and management of Alzheimer's disease (pp. 155-174). London: Martin Dunitz. 
Laws, K. R., Duncan, A., \& Gale, T. M. (2010). "Normal" semanticphonemic fluency discrepancy in Alzheimer's disease? A meta-analytic study. Cortex, 46(5), 595-601.

Lezak, M. D. (1996). Neuropsychological assesment (3 ed.). New York: Oxford University Press.

Lobo, A., Ezquerra, J., Gómez, F. B., Sala, J. M., \& Seva, A. (1979). El miniexamen cognoscitivo. Un test sencillo y práctico para detectar alteraciones intelectuales en pacientes médicos. Actas Luso Españolas de Neurología Psiquiátrica, 7(189-202), 202.

López-Goñi, J. J., Fernández-Montalvo, J., \& Arteaga, A. (2012). Predictive validity of the EuropAsi: Clinical diagnosis or composite scoring? Journal of Substance Abuse Treatment, 42(4), 392-399.

Lorente Aznar, T., Olivera Pueyo, F. J., Benabarre Ciria, S., Rodríguez Torrente, M., Solans Aisa, B., \& Giménez Baratech, A. C. (2010). Rendimiento diagnóstico de los test cognitivos aplicados desde atención primaria. Concordancia y validez de los test de cribado. Atencion primaria / Sociedad Española de Medicina de Familia y Comunitaria, 42(4), 226-232.

Madgison, J. (1989). SPSS/PC+CHAID. Chicago: SPSS Inc.

Marino, J. C., \& Alderete, A. M. (2010). Valores normativos de pruebas de fluidez verbal categoriales, fonológicas, gramaticales y combinadas y análisis comparativo de la capacidad de iniciación. Revista Neuropsicología, Neuropsiquiatría y Neurociencias, 10(1), 82-93.

Moreno, I., Adrián, J. A., Buiza, J. J., \& González, M. (2004). Disponibilidad y fluidez oral de palabras por categorías semánticas: un estudio prospectivo en adultos de más de 55 años con diferente nivel de escolarización. Revista de logopedia, foniatría y fonología, 24(3), 106-118.

Organización Mundial de la Salud. (1992). CIE-10. Trastornos mentales y del comportamiento. Madrid: Meditor.

Pascual, L. F., Fernández, T., López, E., Ríos, C., Martínez, C., González, G., et al. (2000). "Cosas en una casa". Propuesta de tarea alternativa a "animales" en la exploración de la fluidez verbal semántica. Neurología, $15,453$.

Pascual, L. F., Fernández, T., Santos, S., Iñíguez, C., Ríos, C., López, E., et al. (2001). Words generated in the second part of verbal fluency task could be an index of cognitive reserve Journal of Neurology, 248 (suppl), S177.

Pascual, L. F., Fernández, T., Santos, S., Iñíguez, C., Tejero, C., Ríos, C., et al. (2001). Semantic verbal fluency in normal aging Journal of the Neurological Sciences 187 (Suppl), S330.

Pascual Millán, L. F., Martínez Quiñones, J. V., Modrego Pardo, P., Mostacero Miguel, E., López del Val, J., \& Morales Asín, F. (1990). The settest for diagnosis of dementia. Neurologia, 5(3), 82-85.

Peña-Casanova, J., Guardia, J., Bertrán-Serra, I., Manero, R. M., \& Jarne, A. (1997). Versión abreviada del test Barcelona (I): subtest y perfiles normales. Neurología, 12, 99-111.
Peña-Casanova, J., Quiñones-Úbeda, S., Gramunt-Fombuena, N., QuintanaAparicio, M., Aguilar, M., Badenes, D., et al. (2009). Spanish multicenter normative studies (NEURONORMA project): Norms for verbal fluency tests. Archives of Clinical Neuropsychology, 24(4), 395-411.

Peñalva, A., \& López-Goñi, J. J. (2014). Competencias ciudadanas en alumnado de magisterio: la competencia intercultural personal. Revista Electrónica Interuniversitaria de Formación del Profesorado (REIFOP), 17(2), 139153.

Rami, L., Serradell, M., Bosch, B., Villar, A., \& Molinuevo, J. L. (2007). Valores normativos de tests de función cognitiva frontal para la población mayor de 60 años Revista de neurología, 45, 268-271.

Ramírez, M., Ostrosky-Solís, F., Ostrosky-Solís, F., Fernández, A., \& ArdilaArdila, A. (2005). Fluidez verbal semántica en hispanohablantes: Un análisis comparativo. Revista de Neurologia, 41(8), 463-468.

Rosser, A., \& Hodges, J. R. (1994). Initial letter and semantic categoric fluency in Althzeimer's disease, Huntington's disease and Progressive Supranuclear Palsy. Journal of Neurology Neurosurgery and Psychiatry, 57, 13891394.

Sáez-Zea, C., Carnero-Pardo, C., \& Gurpegui, M. (2008). Nombres de personas: una prueba de fluidez verbal sin influencias socioeducativas. Neurología, 23(6), 356-360.

Salorio, P., Navarro, N., Navarro, T., \& López, J. M. (2008). Fluencia verbal y nivel educativo en el deterioro cognitivo. Paper presented at the $9^{\circ}$ Congreso Virtual de Psiquiatría.

Spreen, O., \& Strauss, E. (1998). A compendium of neuropsychological test. Administration, norms and commentary (2 ed.). New York: Oxford.

Steenhuis, R. E., \& Ostbye, T. (1995). Neuropsychological test performance of specific diagnostic groups in the Canadian Study of Health and Aging (CSHA). Journal of Clinical and Experimental Neuropsychology, 17(5), 773-785.

Stern, Y. (2002). Journal of the International Neuropsychological Society. What is cognitive reserve? Theory and research application of the reserve concept, 8 , 448-460.

Tombaugh, T. N., Kozak, J., \& Rees, L. (1999). Normative data stratified by age and education for two measures of verbal fluency: FAS and animal naming. Archives of Clinical Neuropsychology, 14(2), 167-177.

Villodre, R., Sánchez-Alfonso, A., Brines, L., Núñez, A. B., Chirivella, J., Ferri, J., et al. (2006). Fluencia verbal: Estudio normativo piloto según estrategias de «agrupación» $\mathrm{y}$ «saltos» de palabras en población Española de 20 a 49 años. Neurologia, 21(3), 124-130.

(Articulo recibido: 15-02-2013; revisado: 02-04-2013; aceptado: 11-09-2013) 